\title{
Construction of Generalized Integral Formulas By Means of Laplace Transformations
}

\author{
Adam C. Buss, Department of Physics, Indiana University - Northwest \\ Faculty Mentor: Dr. Axel Schulze-Halberg
}

\section{ABSTRACT}

We present a method for the construction of integral identities that contain an undetermined function. Except for mild restrictions, this function can be chosen arbitrarily. Our method is illustrated by several examples leading to new integral identities.

KEYWORDS: generalized integral formula, Laplace transform, definite integral

\section{INTRODUCTION}

$\mathrm{T}$ The closed-form resolution of integrals is one of the standing issues in calculus. Integrals appear in many areas of mathematics and in the natural sciences, particularly in physics and engineering. Existing resources for integral tables, such as the monographs (Abramowitz \& Stegun, 1964; Brychkov, 2008; Gradshtevn \& Ryzhik, 2015) or online data bases (Shapiro, 2015; Wolfram Alpha, 2016), are continuously extended as new methods for the resolution of integrals are discovered. An example for such a new method is presented in the recent work by Glasser (2013). Starting from a known identity, the Laplace transform is used to construct the following integral formula:

$\int_{-\infty}^{\infty} \frac{F\left(x^{2}+i \pi x\right) \cosh (x)}{2+2 \cosh (2 x)} d x=\frac{\pi}{4} F\left(\frac{\pi^{2}}{4}\right)$.

In contrast to the vast majority of identities involving integrals, (1) contains a function $F$ that can be chosen arbitrarily with only slight restrictions. As a result, formula (1) can be used to generate an infinite number of integrals, together with their closed-form resolution. Consequently, generalized integral formulas like (1) are much more versatile than their standard counterparts, such that it is desirable to determine methods for obtaining them. The purpose of the present research is to develop an approach for constructing a class of generalized integral formulas. We start out by considering a group of functions that have a common class of indefinite integrals. By imposing condition on the latter indefinite integrals, we can build integral identities that allow for the construction of generalized integral formulas (Section 2). As a result, in Section 3 we obtain several of such formulas, each of which produces new integral identities.

\section{GENERALIZED INTEGRAL FORMULAS}

In what follows we will first present an example of a generalized integral formula and how to obtain it from an identity that can be found in standard tables. Afterwards, we extend the example, leading to a scheme of construction for generalized integral formulas.

\subsection{GENERALIZED INTEGRAL FORMULAS}

For $t>0$ we consider the following identity (Gradshtevn \& Ryzhik, 2014):

$\int_{0}^{\infty} \frac{\exp [-t \exp (x)]}{1-\exp (-x)}-\frac{2 \exp [-t \exp (2 x)]}{1-\exp (-2 x)} d x=\log (2) \operatorname{cxp}(-t)$

We will now multiply both sides by a continuous function $f$ in the variable $t$. Further restrictions on the properties of $f$ will be developed as our calculation proceeds. After multiplication by $f$ and reordering terms, we obtain:

$\int_{0}^{\infty} \frac{1}{1-\exp (-x)} \exp [-t \exp (x)] f(t)-\frac{2}{1-\exp (-2 x)} \exp [-t \exp (2 x)] f(t) d x=$
$=\log (2) \exp (-t) f(t)$.

In the next step we integrate this relation with respect to the variable $t$ over the interval $(0, \infty)$. Furthermore, we require $f$ to be such that the order of integration can be exchanged. This leads to the result:

$$
\begin{aligned}
& \int_{0}^{\infty} \frac{1}{1-\exp (-x)}\left\{\int_{0}^{\infty} \exp [-t \exp (x)] f(t) d t\right\}- \\
& -\frac{2}{1-\exp (-2 x)}\left\{\int_{0}^{\infty} \exp [-t \exp (2 x)] f(t) d t\right\} d x=\log (2) \int_{0}^{\infty} \exp (-t) f(t) d t .
\end{aligned}
$$

The terms in curly brackets can be interpreted as Laplace transforms of the function $f$. Assuming that $f$ admits such a transform $F$, we can rewrite (4) as follows:

$\int_{0}^{\infty} \frac{F[\exp (x)]}{1-\exp (-x)}-\frac{2 F[\exp (2 x)]}{1-\exp (-2 x)} d x=\log (2) F(1)$

We refer to this identity as a generalized integral formula because it contains a function $F$ that can be chosen arbitrarily, as long as it admits an inverse Laplace transform such that the order of integration in (4) is interchangeable and the integral exists. 


\subsection{GENERALIZATION AND METHOD OF CONSTRUCTION}

Before we proceed, let us formulate four standing assumptions that we will make throughout this work. While these assumptions will be illustrated using our example in Section 2.1, they extend to subsequent considerations in a straightforward manner. First, it is assumed that the function $f$, as it appears in the role of a function that is to be integrated in (3), is continuous. Second, it is assumed that double integrals that arise during the scheme in (4) allow to change their order of integration. Third, it is assumed that all integrals converge, in particular sums or differences of integrals such as in (4). Last, it is assumed that the function $F$ contained in the final result (5) admits an inverse Laplace Transform.

The construction of our integral formula (5) is possible solely due to a particular structure of our initial identity (2). In general, the latter identity must be of the form:

$\int_{0}^{\infty} \sum_{j=1}^{n} A_{j}(x) \exp \left[-t B_{j}(x)\right] d x=C \exp (-D t)$

where $n$ is a natural number, $A_{i}, B_{j}, j=1, \ldots, n$, are functions and $C, D$ denote constants. However, for several reasons relation (6) cannot serve as a starting point when it comes to the practical construction of formulas like (5). This is so because only very particular choices of functions $A_{j}, B_{j}, j=$ $1, \ldots, n$, will permit a closed-form resolution of the integral on the left side of (6). In addition, even if such a resolution is possible, the integral does not necessarily take the form shown on the right side of (6). In order to overcome these issues and construct integral formulas like (5), we will focus on the function that results from indefinite integration on the left side of (6).

For the sake of simplicity let us revisit and analyze our example (2). Indefinite integration gives:

$$
\begin{aligned}
\int \frac{\exp [-t \exp (y)]}{1-\exp (-y)} & -\frac{2 \exp [-t \exp (2 y)]}{1-\exp (-2 y)} d y= \\
& =\exp (-t)\{\operatorname{Ei}[t-t \exp (x)]-\operatorname{Ei}[t-t \exp (2 x)]\}
\end{aligned}
$$

where Ei stands for the exponential integral (Abramowitz \& Stegun, 1964) and a constant of integration was set to zero. Next, we must substitute the integration limits. Starting out with infinity, we make use of the limit relation:

$\lim _{x \rightarrow \infty} \operatorname{Ei}(-x)=0$.

Applying this to the right side of (7) while taking the limit gives:

$$
\begin{aligned}
\lim _{x \rightarrow \infty} \exp (-t)\{\operatorname{Ei}[t-t \exp (x)]-\operatorname{Ei}[t-t \exp (2 x)]\}= & \\
= & =0, \quad t>0 .
\end{aligned}
$$

It remains to evaluate the right side of (7) at $x=0$. Since Ei tends to infinity as its argument approaches zero, we recall the following series expansion:

$\operatorname{Ei}(x)=\log (x)+\gamma+x+O\left(x^{2}\right)$

where $\gamma$ stands for the Euler-Mascheroni constant. On substituting the arguments of the exponential integral given in (9), we obtain:

$$
\begin{aligned}
& \operatorname{Ei}[t-t \exp (x)]-\operatorname{Ei}[t-t \exp (2 x)]= \\
& \quad=\log (t x)-\log (2 t x)+O\left(x^{2}\right)=-\log (2)+O\left(x^{2}\right) .
\end{aligned}
$$

Consequently, we arrive at the expected result:

$$
\begin{gathered}
\lim _{x \rightarrow 0} \exp (-t)\{\operatorname{Ei}[t-t \exp (x)]-\operatorname{Ei}[t-t \exp (2 x)]\}= \\
=\log (2) \exp (-t) .
\end{gathered}
$$

Let us now consider the following function A that generalizes the right side of (7):

$A(x, t)=a_{1}(t) \operatorname{Ei}[b(x, t)]-a_{2}(t) \operatorname{Ei}[c(x, t)]$,

where $a_{1}, a_{2}, b$ and $c$ are functions that are to be determined. We have:

$$
\frac{\partial}{\partial x} A(x, t)=a_{1}(t) \frac{\exp [b(x, t)]}{b(x, t)} \frac{\partial}{\partial x} b(x, t)-a_{2}(t) \frac{\exp [c(x, t)]}{c(x, t)} \frac{\partial}{\partial x} c(x, t)
$$

Now, according to the left side of (6), we want each of the terms in curly brackets to be a product of an exponential and a factor that is independent of the variable $t$. Since we already have an exponential in both terms, it is reasonable to require:

$$
\frac{\partial}{\partial t} \frac{1}{b(x, t)} \frac{\partial}{\partial x} b(x, t)=0 \quad \frac{\partial}{\partial t} \frac{1}{c(x, t)} \frac{\partial}{\partial x} c(x, t)=0 .
$$

These conditions are fulfilled if the following choice for $b$ and $c$ is employed:

$$
b(x, t)=b_{1}(l) b_{2}(x) \quad c(x, l)=c_{1}(l) c_{2}(x) .
$$

where $b_{j}$ and $c_{i}, j=1,2$, are functions depending on a single variable that will be determined further. Upon substituting (12) into (11), we obtain:

$A(x, l)=a_{1}(l) \operatorname{Ei}\left[b_{1}(l) b_{2}(x)\right]-a_{2}(l) \operatorname{Ei}\left[c_{1}(l) c_{2}(x)\right]$,

and:

$$
\begin{aligned}
\frac{\partial}{\partial x} A(x, t) & = \\
& =a_{1}(t) \exp \left[b_{1}(t) b_{2}(x)\right] \frac{b_{2}^{\prime}(x)}{b_{2}(x)}-a_{2}(t) \exp \left[c_{1}(t) c_{2}(x)\right] \frac{c_{2}^{\prime}(x)}{c_{2}(x)} .
\end{aligned}
$$


Comparison of this expression with the integrand on the left side of (6) shows that the functions $a_{1}, a_{2}$, can be chosen as follows:

$$
\begin{aligned}
a_{1}(l)=\exp \left(-k_{1} l\right) & a_{2}(l)=\exp \left(-k_{2} l\right) \\
b_{1}(t)=k_{3} t & c_{1}(l)=k_{4} t,
\end{aligned}
$$

where $k_{j}, j=1, \ldots, 4$, are constants. These settings render (13) in the form:

$$
\begin{aligned}
A(x, t) & =\exp \left(-k_{1} \iota\right) \operatorname{Ei}\left[k_{3} \iota b_{2}(x)\right]- \\
& -\exp \left(-k_{2} \iota\right) \operatorname{Ei}\left[k_{4} l c_{2}(x)\right] .
\end{aligned}
$$

while for its partial derivative (14) we find:

$$
\frac{\partial}{\partial x} A(x, t)=\exp \left[\left(k_{3}-k_{1}\right) t b_{2}(x)\right] \frac{b_{2}^{\prime}(x)}{b_{2}(x)}-\exp \left\{\left(k_{4}-k_{2}\right) t c_{2}(x)\right] \frac{c_{2}^{\prime}(x)}{c_{2}(x)} .
$$

In the next step we will substitute the limits of integration into (15). Starting out with infinity, we take our identity (8) into account. This gives the constraints:

$$
\lim _{x \rightarrow \infty} k_{3} b_{2}(x)=-\infty \quad \lim _{x \rightarrow \infty} k_{4} c_{2}(x)=-\infty .
$$

Taking the limit of (15) at zero is more complicated because the behavior of the functions $b_{2}$ and $c_{2}$ at zero is not known. While we are unable to give a general criterion for the limit to exist, in the example section we will discuss typical scenarios that lead to a finite limit.

\section{APPLICATIONS}

We will first take our previous formula (2) and evaluate it for several particular cases. Afterwards, we construct a new generalized integral formula (15). In our final example we show that the latter indefinite integral can also be used to build formulas involving complex functions.

\subsection{EXPONENTIAL AND HYPERBOLIC INTEGRANDS}

We revisit our integral formula (2), which can be obtained by comparison of the indefinite integrals (7) and (15). Both coincide if the following settings are employed:

$k_{1}=k_{2}=k_{3}=k_{4}=1$

$b_{2}(x)=1-\exp (x) \quad c_{2}(x)=1-\exp (2 x)$.

Let us now state a few particular cases of the generalized integral formula (5) resulting from (15) with the settings (17). Recall that we must choose the function F such that it admits an inverse Laplace transform and such that the resulting integral exists. Starting out with a simple example, we plug $\mathrm{F}(x)=1 / x$ into (5), which gives:

$$
\int_{0}^{\infty} \frac{1}{1+\exp (x)} d x=\log (2)
$$

Another simple example is generated if we choose $\mathrm{F}(x)=1 /(x$ $+1)$. We obtain after conversion of exponentials to hyperbolic functions:

$$
\int_{0}^{\infty} \operatorname{sech}(x) \tanh \left(\frac{x}{2}\right) d x=\log (2) .
$$

Let us now generate a less elementary integral relation. Upon setting $\mathrm{F}(\mathrm{x})=\operatorname{erf}(1 / x)$, where erf denotes the error function (Abramowitz \& Stegun, 1964), we get from (5):

$\int_{0}^{\infty} \frac{\exp (x)}{\operatorname{cxp}(2 x)-1}\{[\exp (x)+1] \operatorname{erf}[\exp (-x)]-2 \exp (x) \operatorname{erf}[\exp (-2 x)]\} d x=\log (2) \operatorname{erf}(1)$.

Before we conclude this example, let us remark that our integral formula (2) can be generalized further if we leave the constants $k_{\mathrm{j}}, j=1, \ldots 4$, arbitrary.

\subsection{LOGARITHMIC INTEGRANDS}

Let us now make the following settings in (15):

$$
\begin{aligned}
k_{2}= & k_{1} \\
& c_{2}(x)=-\log (x+1),
\end{aligned}
$$

where we further assume that $k_{1}, k_{3}, k_{4}>0$. After substitution, (15) takes the form:

$A(x, t)=\exp \left(-k_{1} t\right)\left\{\operatorname{Ei}\left(-k_{3} t x\right)-\operatorname{Ei}\left[-k_{4} t \log (x+1)\right]\right\}$

The partial derivative (14) of this function is given by:

$\frac{\partial}{\partial x} A(x, t)=\frac{1}{x} \exp \left[-t\left(k_{1}+k_{3} x\right)\right]-\frac{\exp \left(-k_{1} t\right)}{(x+1)^{k_{4} t+1} \log (x+1)}$.

Keeping this in mind, in the next step we substitute the limits of integration into the function (18). Starting with infinity, we make use of (16) in order to obtain:

$$
\lim _{x \rightarrow \infty} A(x, t)=0, \quad t>0 .
$$

Next, we observe that the arguments of both exponential integrals in (18) vanish as $x$ tends to zero, such that (10) becomes applicable. We get after some simplification:

$\lim _{x \rightarrow 0} A(x, t)=\log \left(\frac{k_{4}}{k_{3}}\right) \exp \left(-k_{1} t\right), \quad t>0$.

Now, combination of (19) and (20) gives the following identity for $t>0$ :

$\int_{0}^{\infty} \frac{1}{x} \exp \left[-t\left(k_{1}+k_{3} x\right)\right]-\frac{\exp \left(-k_{1} t\right)}{(x+1)^{k_{4} t+1} \log (x+1)} d x=\log \left(\frac{k_{4}}{k_{3}}\right) \exp \left(-k_{1} t\right)$. 
We can now apply the same procedure as in our first example, that is, multiply (21) by a function $f$ depending on the variable $t$, and integrate over the interval $(0, \infty)$. Assuming that $f$ is such as to allow an exchange of the integration order and to admit a Laplace transform, we arrive at the generalized integral formula:

$\int_{0}^{\infty} \frac{F\left(k_{1}+k_{3} x\right)}{x}-\frac{F\left[k_{1}+k_{4} \log (x+1)\right]}{(x+1) \log (x+1)} d x=\log \left(\frac{k_{4}}{k_{3}}\right) F\left(k_{1}\right)$.

Recall that this relation holds as long as $F$ has an inverse Laplace transform. Let us now present a few particular cases of (22) for different choices of the function $F$. For the sake of brevity, we first apply the overall settings $k_{1}=4, k_{3}=1$ and $k_{4}$ $=2$. Picking $\mathrm{F}(x)=1 / x$ renders (22) in the form:

$\int_{0}^{\infty} \frac{F\left(k_{1}+k_{3} x\right)}{x}-\frac{F\left[k_{1}+k_{4} \log (x+1)\right]}{(x+1) \log (x+1)} d x=\log \left(\frac{k_{4}}{k_{3}}\right) F\left(k_{1}\right)$.

Next, we plug $\mathrm{F}(x)=\exp (4-x)$ into our formula (22). We obtain:

$$
\int_{0}^{\infty} \frac{1}{x^{2}+4 x}-\frac{1}{2(x+1) \log (x+1)[\log (x+1)+2]} d x=\frac{\log (2)}{4} .
$$

Note that the right side does not change because we did not modify the constants $k_{1}, k_{3}$ and $k_{4}$.

\subsection{COMPLEX INTEGRANDS}

Except for the introductory formula (1), throughout the preceding considerations we assumed that the indefinite integral (15) and the function it contains are real. In particular, our calculations of the integration limits are based on real-valued functions. In this section we will show that choosing complex functions $b_{2}$ and $c_{2}$ in (15) can lead to integral formulas that extend those previously studied here. It is important to point out that our criterion (16) for determining the limit of A at infinity does not hold anymore if $b_{2}$ and $c_{2}$ are complex-valued. While a general analysis of this case is beyond the scope of this note, we will present an example. Starting out from the indefinite integral (15), making the following settings:

$$
\begin{array}{cr}
k_{1}=k_{3}=k_{4}=1 & k_{2}=-1 \\
b_{2}=1-i x & c_{2}=-1-i x .
\end{array}
$$

This renders our function (15) in the form:

$$
A(x, \iota)=\exp (-\iota) \operatorname{Ei}[-l(-1+i x)]-\exp (\iota) \operatorname{Ei}[-\iota(1+i x)] .
$$

Its partial derivative (14) becomes:

$\frac{\partial}{\partial x} A(x, t)=-2 i \frac{\exp (-i t x)}{x^{2}+1}$
We now evaluate the limit of (23) if $x$ tends to infinity. We obtain:

$$
\lim _{x \rightarrow \infty} A(x, t)=-\frac{i \pi}{2}[\exp (-t)+\exp (t)]
$$

Instead of taking zero as the lower limit of integration, this time we will use negative infinity:

$\lim _{x \rightarrow-\infty} A(x, t)=-\frac{i \pi}{2}[-3 \exp (-t)+\exp (t)]$.

Upon combining (24) and (25), (26) we get after multiplication by $i / 2$ the relation:

$$
\int_{-\infty}^{\infty} \frac{\exp (-i t x)}{x^{2}+1} d x=\pi \exp (-t)
$$

The form of this identity is suitable for the construction of a generalized integral formula. Multiplication of both sides by a function $f$ that has a two-sided Laplace transform $F$ and exchanging the order of integration yields:

$$
\int_{-\infty}^{\infty} \frac{F(i x)}{x^{2}+1} d x=\pi F(1)
$$

Before we state particular cases of this identity, let us remark that due to the complex argument of $F$ and the domain of integration being the whole real line, the choices for $F$ are much more restricted than in the previous examples. As mentioned above, we will not go into details, but just evaluate (27) for a few cases. The simple setting $\mathrm{F}(x)=1$ gives the known relation:

$$
\int_{-\infty}^{\infty} \frac{1}{x^{2}+1} d x=\pi
$$

Next, we choose $\mathrm{F}(x)=x^{2} /(x+1)^{2}$, which renders (27) in the form:

$$
\int_{-\infty}^{\infty} \frac{x^{2}}{(x-i)^{3}(x+i)} d x=\frac{\pi}{4}
$$

Finally, let us substitute $\mathrm{F}(x)=\arctan \left(x^{4}\right)$ into (27). We obtain the result:

$$
\int_{-\infty}^{\infty} \frac{\arctan \left(x^{4}\right)}{x^{2}+1} d x=\frac{\pi^{2}}{4}
$$

As mentioned above, we restrict ourselves to the complex integral formula (27) because the criteria for choosing the function $\mathrm{F}$ become much more complicated than in the real case. 


\section{CONCLUDING REMARKS}

In this note we have presented a simple method for the construction of generalized integral formulas containing an almost arbitrary function rather than numerical parameters.

It should be stressed that our method can be generalized by replacing the Laplace transform through a different integral transform, such as the bilateral Laplace transform or the Fourier transform. This broadens the choice of functions in the resulting generalized integral formula and it also allows to modify the domain of integration.

\section{AUTHOR INFORMATION}

All correspondence should be sent to acbuss@iun.edu.

\section{ACKNOWLEDGMENTS}

The first author acknowledges funding through the Indiana University Northwest Undergraduate Research Fund. The first author would also like to thank Dr. Axel Schulze-Halberg for his mentorship and support through the various stages of this research project.

\section{REFERENCES}

Abramowitz, M., \& Stegun, I. (1964). Handbook of Mathematical Functions with Formulas, Graphs, and Mathematical Tables. Mineola, NY: Dover Publications.

Brychkov, Y.A. (2008). Handbook of Special Functions; Derivatives, Integrals, Series and Other Formulas. Boca Raton, FL: CRC Press.

Glasser, M.L. (2013). A remarkable property of definite integrals. Mathematics of Computation, 40(162), 561563.

Gradshteyn, I.S., \& Ryzhik, I.M (2014). Tables of integrals, series, and products. In D. Zwillinger \& V. Moll (Eds.) Tables of Integrals, Series, and Products. Waltham, MA: Academic Press.

Shapiro, B.E. (2015). Table of Integrals. Retrieved from: http://integral-table.com

Wolfram Alpha (2016). Wolfram Alpha: Computational Knowledge Engine. Retrieved from: https://www. wolframalpha.com 